\title{
p-Channel and n-Channel Thin-Film-Transistor Operation on Sprayed ZnO Nanoparticle Layers
}

\author{
Daiki Itohara, Kazato Shinohara, Toshiyuki Yoshida, and Yasuhisa Fujita \\ Interdisciplinary Graduate School of Science and Engineering, Shimane University, 1060 Nishikawatsucho, Matsue 690-8504, Japan \\ Correspondence should be addressed to Toshiyuki Yoshida; yosisi@ecs.shimane-u.ac.jp
}

Received 26 April 2016; Accepted 3 July 2016

Academic Editor: Antonios Kelarakis

Copyright (C) 2016 Daiki Itohara et al. This is an open access article distributed under the Creative Commons Attribution License, which permits unrestricted use, distribution, and reproduction in any medium, provided the original work is properly cited.

\begin{abstract}
Both $\mathrm{n}$-channel and $\mathrm{p}$-channel thin-film transistors have been realized on $\mathrm{ZnO}$ nanoparticle (NP) layers sprayed onto quartz substrates. In this study, nitrogen-doped $\mathrm{ZnO}$-NPs were synthesized using an arc-discharge-mediated gas-evaporation method that was recently developed. Sprayed NP layers were characterized by scanning electron microscopy and Hall effect measurements. It was confirmed that p-type behaving NP layers can be obtained using ZnO-NPs synthesized with lower chamber pressure, whereas n-type conductivity can be obtained with higher chamber pressure. pn-junction diodes were also tested, resulting in clear rectifying characteristics. The possibility of particle-process-based $\mathrm{ZnO}-\mathrm{NP}$ electronics was confirmed.
\end{abstract}

\section{Introduction}

Zinc oxide $(\mathrm{ZnO})$ attracts considerable attention because it is an oxide semiconductor with a direct wide bandgap of $3.37 \mathrm{eV}$ at room temperature, it can be developed for largearea substrates, and it exhibits high-field effect mobility. Due to these advantages, $\mathrm{ZnO}$ is a promising candidate for applications to optoelectronic devices such as light-emitting diodes (LEDs) [1] and laser diodes, as well as to electronic devices such as thin-film transistors (TFTs) [2-6].

One difficulty faced by $\mathrm{ZnO}$ application is that it is challenging to obtain p-type conductivity with this material because of the compensation by intrinsic donor defects such as oxygen vacancies and $\mathrm{Zn}$ interstitials [7, 8]. Various approaches have been reported for achieving p-type $\mathrm{ZnO}$ layers, like doping with group Ia [9, 10], Ib [11], and V [12-15] elements, codoping with group Ia, III, or V elements [16, 17], and so on $[18,19]$. However, few results can achieve LED or TFT operations using the obtained p-type layers.

We recently succeeded in synthesizing nitrogen-doped $\mathrm{ZnO}$ nanoparticles (NPs) using a gas-evaporation method. This method was confirmed to p-type conductivity using the scanning nonlinear dielectric microscopy (SNDM) technique [20]. Fujita et al. [21] reported pn-junction-type nearultraviolet (UV) LEDs using these NPs on Ga-doped $\mathrm{ZnO}$ (GZO) films. These results suggest that doped nitrogen atoms are incorporated into oxygen sites, acting successfully as acceptors [21-25]. By regulating the conditions of the gasevaporation method, $\mathrm{ZnO}$-NPs with n-type conductivity can also be obtained [20]. These results strongly indicate that both p-type and n-type $\mathrm{ZnO}$-NP layers can be obtained, allowing the achievement of $\mathrm{p}$ - and n-channel ZnO-TFTs. Previously, some approaches were developed to form TFTs using $\mathrm{ZnO}$ NP layers [26-29]; however, only n-type conductive NP layers succeeded for TFT operations.

In this study, we attempt to realize both $\mathrm{p}$ - and n-type ZnO-NP layers on glass (quartz) substrates using a spray method. Then, we present the first ever demonstration of $\mathrm{p}$ and n-channel depletion-type TFTs.

\section{Experimental}

$\mathrm{ZnO}$-NPs were synthesized by arc-discharge-mediated gas evaporation using dry air, as shown in Figure 1(a). By this method, $\mathrm{Zn}$ metal was evaporated by direct current (DC) arc discharge and oxidized in the supplied dry air. Incorporation and doping of nitrogen atoms at $\mathrm{O}$-sites in $\mathrm{ZnO}-\mathrm{NPs}$ were performed by $\mathrm{N}_{2}$ radicals generated by arc plasma. Thus, extending the lifetime of $\mathrm{N}_{2}$ radicals and increasing the number of $\mathrm{O}$ vacancies were effective means of nitrogen doping. The low chamber pressure led to the lifetime extension of $\mathrm{N}_{2}$ radicals through fewer collisions, and the 


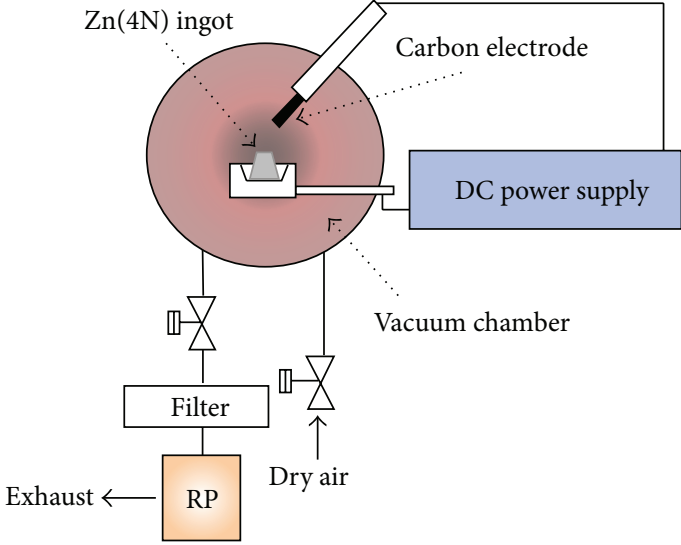

(a)

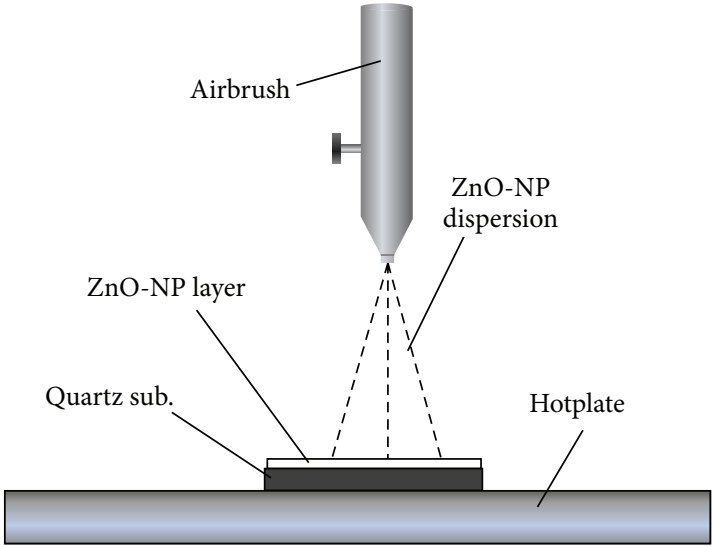

(b)

FIGURE 1: Schematic illustrations of (a) the gas-evaporation method and (b) the spray method.

high arc current caused excess evaporation of $\mathrm{Zn}$, leading to deficiency of oxygen. In our evaporation system, a chamber pressure of 150 Torr and an arc current of $50 \mathrm{~A}$ were suitable for enhanced nitrogen doping (referred to as "type A"); on the other hand, 610 Torr and $30 \mathrm{~A}$ were chosen for suppressed nitrogen doping (referred to as "type B"), in the expectation of obtaining p-type and n-type $\mathrm{ZnO}$-NPs, respectively.

The dispersions used for the spray process were prepared by mixing and dispersing $0.2 \mathrm{~g}$ of $\mathrm{ZnO}-\mathrm{NPs}$ and $20 \mathrm{~g}$ of water using an ultrasonic homogenizer with power of $150 \mathrm{~W}$ for $3 \mathrm{~min}$ and by centrifuging with a force of $3,000 \mathrm{G}$ for $1 \mathrm{~min}$ to remove large-sized NPs. Particle size distributions using type A and type B NPs were similar to the median size of $200 \mathrm{~nm}$.

The NP layers were formed on the quartz substrates by a spray method, as shown in Figure 1(b). $30 \mathrm{~mL}$ of dispersion fluid was sprayed over 500 bursts with $5 \mathrm{~s}$ intervals. The hotplate temperatures were $250^{\circ} \mathrm{C}$ and $500^{\circ} \mathrm{C}$ for dispersions with type $\mathrm{A}$ and type B NPs, respectively. To avoid desorption of the nitrogen atoms from NPs, a reduced temperature was applied for the former ones.

Morphologies were examined by field-emission scanning electron microscopy (FESEM; JSM-7001FA, JEOL, $5 \mathrm{kV}$ ). The sheet resistance was determined independently by the transfer length method (TLM) for electrode distances of 100 to $500 \mu \mathrm{m}$, and the electronic mobility and carrier concentration were evaluated by the van der Pauw method (HL5500PC with HL5580 Buffer Amplifier, ACCENT). For ohmic electrodes, based on their different work functions, $\mathrm{Au}$ and $\mathrm{Al}$ were used for the layers using type A and type B NPs, respectively.

\section{Results and Discussions}

Figure 2(a) shows the typical cross-sectional SEM image for the obtained $\mathrm{ZnO}$-NP layer, indicating that the thickness of the obtained NP layer was about $20 \mu \mathrm{m}$, which agrees with the value calculated using the sprayed area and the total volume of the sprayed particles. Figures 2(b) and 2(c) show the top view of the sprayed surfaces with type A and type B NPs, respectively. Both images indicate that the quartz

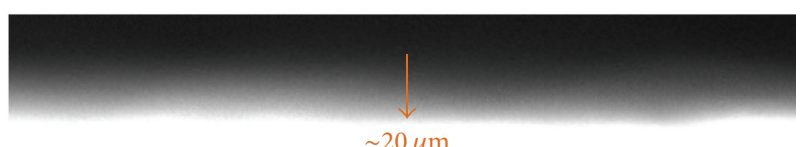

$\sim 20 \mu \mathrm{m}$

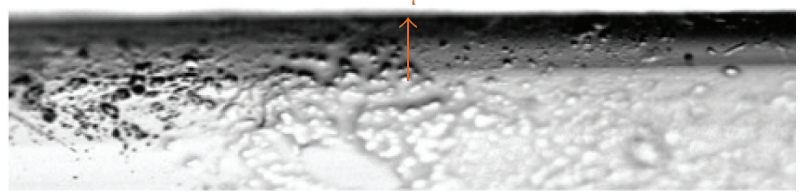

(a)

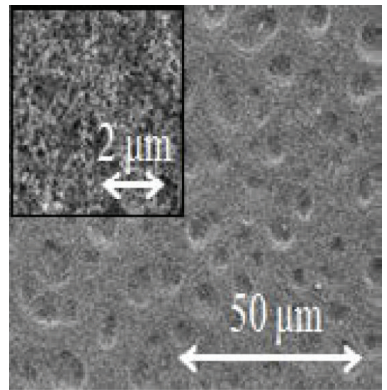

(b)

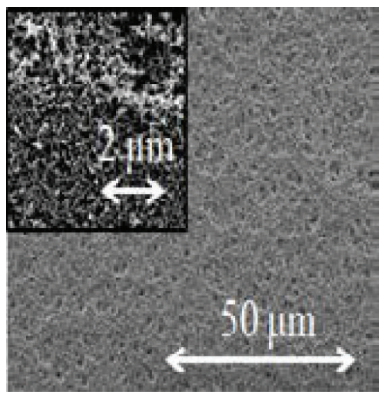

(c)
FIGURE 2: (a) Cross-sectional SEM images of the ZnO-NP layer. SEM top view images for sprayed (b) type A and (c) type B NP layers.

surfaces were covered with NPs with sufficient uniformity. Figure 2(b) shows relatively rough morphology, since the hotplate temperature was lower and it needed a relatively long time to evaporate, leading to cohesion phenomenon.

The Hall effect measurement results for NP layers using type A and type B NPs are summarized in Table 1. A notable result is the carrier polarity shown in the signs of the carrier concentrations. Especially in the values for type A NP layers, signs of plus were obtained reproducibly. Furthermore, another behavioral analysis between the carrier concentration (sign and quantity) and the content of nitrogen atoms, which were examined using $\mathrm{ZnO}-\mathrm{NPs}$ synthesized with different arc current and chamber pressure, showed 


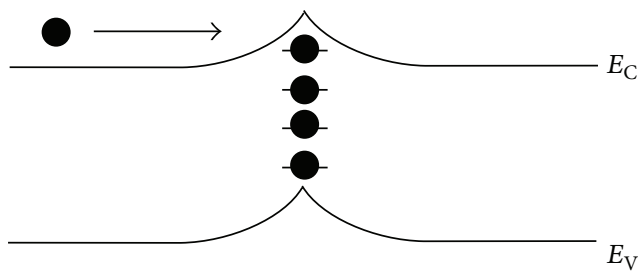

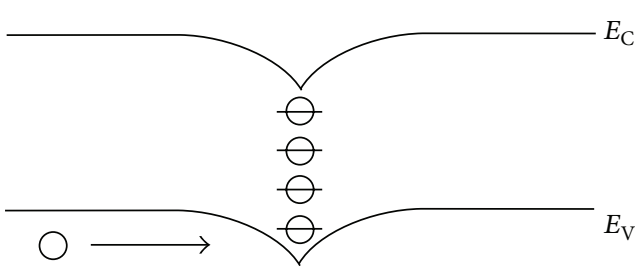

- Electron ○ Hole

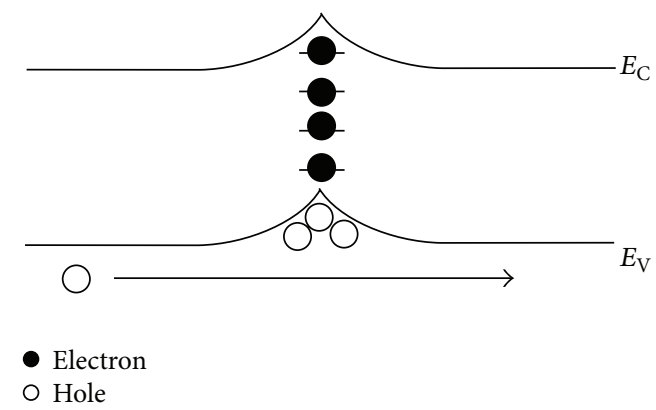

(b)

FIgURE 3: Carrier trapped conditions and band diagrams around GB regions for (a) the usual cases and (b) the acceptor type traps.

TABLE 1: Hall measurement results.

\begin{tabular}{lcc}
\hline Parameters & Type A & Type B \\
\hline $\begin{array}{l}\text { Mobility } \\
\left(\mathrm{cm}^{2} / \mathrm{Vs}\right)\end{array}$ & $5.04 \sim 6.98$ & $0.18 \sim 0.74$ \\
$\begin{array}{l}\text { Carrier } \\
\text { concentration } \\
\left(\mathrm{cm}^{-3}\right)\end{array}$ & $+2.0 \times 10^{12} \sim+2.7 \times 10^{12}$ & $-7.0 \times 10^{15} \sim-5.5 \times 10^{16}$ \\
$\begin{array}{l}\text { Sheet resistance } \\
(\Omega / \square)\end{array}$ & $2.3 \times 10^{9}$ & \\
\hline
\end{tabular}

significant relationship; that is, high nitrogen content yielded a plus sign and low content yielded a minus sign (not shown here). From these results, it can be guessed that the type A NP layers have p-type conductivity. The mobility of type A NP layers (hole) was larger than the mobility of type B ones (electron), whereas the hole mobility is smaller than the electron mobility in usual single crystal $\mathrm{ZnO}$ [19]. Recent nitrogen-doped $\mathrm{ZnO}-\mathrm{NP}$ layers containing grain boundaries (GBs) show similar behaviors [13-15]. Generally, the GBs affect the carrier transfer, since the majority carriers will be trapped at the defects in GBs' site with forming energy barriers as shown in Figure 3(a). But, in p-ZnO-NP layers, if the electrons were trapped at the defects in GBs' site, hole transfer may not be affected by GBs (as shown in Figure 3(b)). One report [30] indicates that the excess oxygen at the GB area acts as acceptor, capturing the electrons and assisting the hole transfer. It was confirmed that there was $\mathrm{OH}$ group at the surface of $\mathrm{ZnO}-\mathrm{NPs}$ used in this study [31], which may contribute to electron trapping at GB site and behave similarly as shown in Figure 3(b). Another approach to explain the higher hole mobility compared to electron one in the NP layers (quantum dot films) was provided in the recent literature [32], applying the variable-range hopping model to the carrier transfer from one NP to neighboring NPs. In this calculation, NP layers with size dispersions can be determined using molecular dynamics simulations. For the random distribution of various NP sizes in the film, which is suitable for $\mathrm{ZnO}$-NP layers used in this study, the resultant degradation of the electron mobility increased with the increase of the size dispersion, while the hole mobility showed less degradation. Since the size dispersions of $\mathrm{ZnO}$ NPs used in this study are large enough compared to those in these calculations, larger degradation of electron mobility than that of hole mobility should occur, leading to higher mobility in type A NP layers. Here, it is not clear yet which model (i.e., enlarging the hole mobility by the electrons in GBs or the degradation of the electron mobility by largesize dispersions of NPs) is true; however, the reproducible larger mobility values can be evidence for p-type conduction in type A ZnO-NP layers. On the other hand, the low carrier concentration and the high sheet resistance values indicate that further investigations and process optimizations are required. In this paper, for convenience, the layers formed using type A and type B NPs are described as p-type and ntype layers, respectively.

By stacking the p-type ZnO-NP layer on the n-type one, pn-junction diodes were fabricated on quartz substrates, as schematically shown in the inset of Figure 4(a). The spraying conditions for each of the n-type and p-type layers were the same as described above. The obtained $I-V$ characteristic is shown in Figure 4(a), indicating some rectifying characteristics. To avoid Schottky barrier formation, ohmic behaviors were examined individually on $\mathrm{n}$ - and p-type layers, as shown in Figures 4(b) and 4(c), respectively. Obvious ohmic contacts were confirmed at the contacting electrodes on each $\mathrm{n}$ - and p-type $\mathrm{ZnO}-\mathrm{NP}$ region. Thus, the 


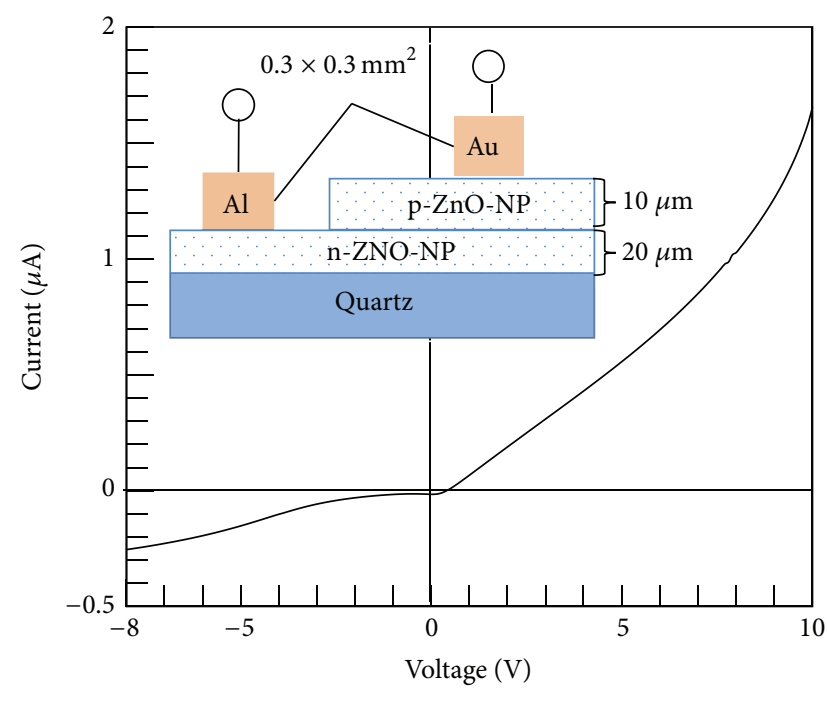

(a)

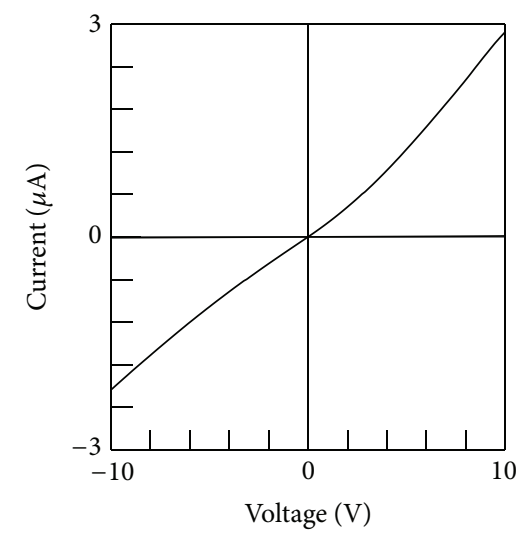

(b) n-type

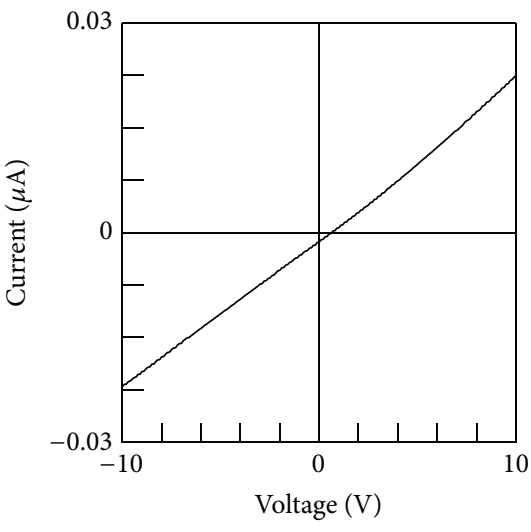

(c) p-type

FIGURE 4: (a) $I-V$ characteristic of the pn-junction diode studied in this paper. The cross-sectional illustration is in the inset. Ohmic tests for the contact electrodes on (b) n-type and (c) p-type ZnO-NP layers, respectively.

rectifying behavior shown in Figure 4(a) was confirmed to be obtained from the pn-junction structure. However, the leakage current in the reverse bias region was relatively high; in synthesizing the p-type $\mathrm{ZnO}$-NPs, an inhomogeneous arc plasma density distribution may have caused the formation of fewer nitrogen-doped or n-type NPs, whose possibility was discussed in [21]. One possible reason for the leakage current in the reverse bias region is the existence of n-type NPs in the p-type layer. Another problem is that the turn-on voltage in the forward bias region was smaller than about $3 \mathrm{~V}$, which was expected from the $\mathrm{ZnO}$ bandgap energy. The conductive mechanism in the NP film may affect this point; however, further investigation is required.

Back gate metal-oxide-semiconductor depletion-type TFTs were fabricated on $\mathrm{Si} / \mathrm{SiO}_{2}$ substrates using the sprayed p-type or n-type $\mathrm{ZnO}$-NP layers obtained in this study. The schematic cross-sectional device structure was shown in Figure 5(a). The thicknesses of $\mathrm{Si} / \mathrm{SiO}_{2} / \mathrm{ZnO}-\mathrm{NP}$ were $500 \mu \mathrm{m} / 0.078 \mu \mathrm{m} / 20 \mu \mathrm{m}$, respectively. Au and $\mathrm{Al}$ electrodes were patterned on p-type and n-type layers, respectively, as source/drain contacts. The distance between source and drain (gate length) was $450 \mu \mathrm{m}$, and the width was $300 \mu \mathrm{m}$. Figures 5(b) and 5(c) show the $I_{D}-V_{D}$ curves of TFTs using p-type and n-type $\mathrm{ZnO}$-NP layers, showing the decrease in drain currents with the positive and negative back gate biases, respectively. It has been confirmed that transistor behaviors were obtained using each p-type or n-type $\mathrm{ZnO}$-NP layer as a channel region. Transistor performances and stabilities are insufficient for the practical use in this stage; however, we have demonstrated the world's first p-type and n-type ZnO-based TFTs using NP-deposition layers. The possibility of future contributions to extremely simple and low-cost NP and spray processes for $\mathrm{ZnO}-\mathrm{NP}$-based electronics has been confirmed.

\section{Conclusions}

ZnO-NP layers were successfully obtained by spraying dispersions of $\mathrm{ZnO}-\mathrm{NPs}$, which can be an extremely simple and low-cost process. ZnO-NPs were synthesized using an arc-discharge-mediated gas-evaporation method, providing nitrogen-doped ZnO-NPs. Depending on the synthesizing conditions, sprayed NP layers showed p-type and n-type conductivities with high reproducibility. Discussions about the reason for higher mobility in p-type layers than that in ntype ones are added, which can support the fact of realization of the p-type $\mathrm{ZnO}-\mathrm{NP}$ layers. pn-junction behaviors were tested using these layers, showing clear rectifying characteristics. Finally, operations of p-channel and n-channel TFTs on sprayed $\mathrm{ZnO}-\mathrm{NP}$ layers used in this study were successfully 


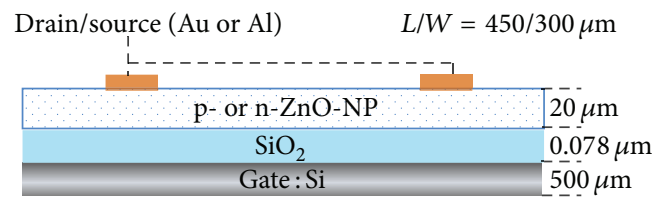

(a)

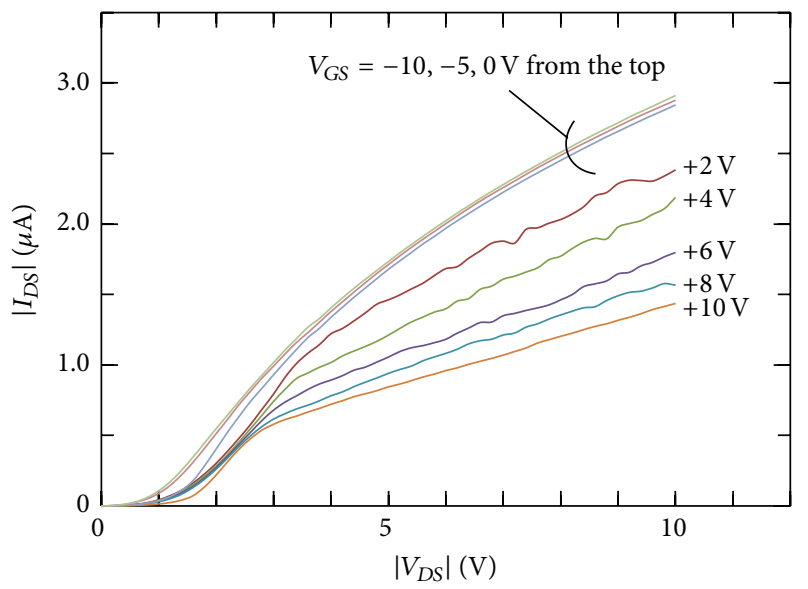

(b)

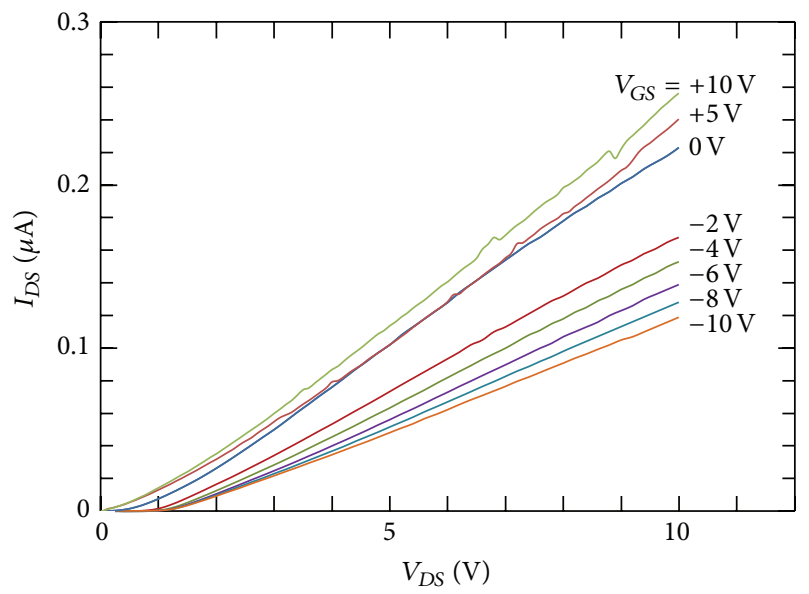

(c)

FIGURE 5: (a) Schematic illustration of back gate thin-film transistor formed on $\mathrm{Si} / \mathrm{SiO}_{2}$ substrate. The characteristics of $I_{D S}$ versus $V_{D S}$ of TFTs with channel regions using (b) p-type and (c) n-type ZnO-NP layers.

demonstrated. These results are expected to lead to further contributions to the future $\mathrm{ZnO}-\mathrm{NP}$-based electronics.

\section{Competing Interests}

The authors declare that there are no competing interests regarding the publication of this paper.

\section{Acknowledgments}

The authors acknowledge the cooperation of the Center for Integrated Research in Science, Shimane University, for providing the experimental facility of FESEM, which was introduced through Tatara Project supported by the Ministry of Education, Culture, Sports, Science and Technologies of Japan. This work was supported by JSPS KAKENHI Grant no. 25870448.

\section{References}

[1] Y.-S. Choi, J.-W. Kang, D.-K. Hwang, and S.-J. Park, "Recent advances in $\mathrm{ZnO}$-based light-emitting diodes," IEEE Transactions on Electron Devices, vol. 57, no. 1, pp. 26-41, 2010.

[2] J. Zhu, H. Chen, G. Saraf, Z. Duan, Y. Lu, and S. T. Hsu, “ZnO TFT devices built on glass substrates," Journal of Electronic Materials, vol. 37, no. 9, pp. 1237-1240, 2008.

[3] L. Zhang, H. Zhang, Y. Bai et al., "Enhanced performances of ZnO-TFT by improving surface properties of channel layer," Solid State Communications, vol. 146, no. 9-10, pp. 387-390, 2008.

[4] J. H. Chung, J. Y. Lee, H. S. Kim, N. W. Jang, and J. H. Kim, "Effect of thickness of $\mathrm{ZnO}$ active layer on ZnO-TFT's characteristics," Thin Solid Films, vol. 516, no. 16, pp. 5597-5601, 2008.

[5] R. Navamathavan, C. K. Choi, E.-J. Yang, J.-H. Lim, D.-K. Hwang, and S.-J. Park, "Fabrication and characterizations of $\mathrm{ZnO}$ thin film transistors prepared by using radio frequency magnetron sputtering," Solid-State Electronics, vol. 52, no. 5, pp. 813-816, 2008.

[6] N. Münzenrieder, P. Voser, L. Petti et al., "Flexible self-aligned double-gate IGZO TFT," IEEE Electron Device Letters, vol. 35, no. 1, pp. 69-71, 2014.

[7] A. Lajn, H. Von Wenckstern, G. Benndorf et al., "Shallow donors and compensation in homoepitaxial $\mathrm{ZnO}$ thin films," Journal of Electronic Materials, vol. 39, no. 5, pp. 595-600, 2010.

[8] K. Iwata, P. Fons, A. Yamada, K. Matsubara, and S. Niki, "Nitrogen-induced defects in $\mathrm{ZnO}: \mathrm{N}$ grown on sapphire substrate by gas source MBE," Journal of Crystal Growth, vol. 209, no. 2-3, pp. 526-531, 2000.

[9] L. Tanga, B. Wang, Y. Zhang, and Y. Gu, "Structural and electrical properties of Li-doped p-type $\mathrm{ZnO}$ thin films fabricated by RF magnetron sputtering," Materials Science and Engineering $B$, vol. 176, no. 7, pp. 548-551, 2011.

[10] Y. F. Lu, K. W. Wu, Y. J. Zeng et al., "Local p-type conduction of Na-doped ZnO thin films grown by MOCVD," Chemical Physics Letters, vol. 582, pp. 82-85, 2013.

[11] M. Suja, S. B. Bashar, M. M. Morshed, and J. Liu, "Realization of $\mathrm{Cu}$-doped p-type $\mathrm{ZnO}$ thin films by molecular beam epitaxy," ACS Applied Materials \& Interfaces, vol. 7, no. 16, pp. 8894-8899, 2015.

[12] Y. Ma, Q. Gao, G. G. Wu et al., "Growth and conduction mechanism of As-doped p-type $\mathrm{ZnO}$ thin films deposited by MOCVD," Materials Research Bulletin, vol. 48, no. 3, pp. 12391243, 2013.

[13] L.-C. Chao, J.-W. Chen, H.-C. Peng, and C.-H. Ho, "Characterization of nitrogen doped p-type $\mathrm{ZnO}$ thin films prepared by reactive ion beam sputter deposition," Surface and Coatings Technology, vol. 231, pp. 492-495, 2013.

[14] X. Nie, B. Zhang, J. Wang, L. Shi, Z. Di, and Q. Guo, "Roomtemperature ferromagnetism in p-type nitrogen-doped $\mathrm{ZnO}$ films," Materials Letters, vol. 161, pp. 355-359, 2015.

[15] T. K. Pathak, V. Kumar, H. C. Swart, and L. P. Purohit, "Effect of doping concentration on the conductivity and optical 
properties of p-type $\mathrm{ZnO}$ thin films," Physica B: Condensed Matter, vol. 480, pp. 31-35, 2016.

[16] M. Joseph, H. Tabata, H. Saeki, K. Ueda, and T. Kawai, "Fabrication of the low-resistive p-type $\mathrm{ZnO}$ by codoping method," Physica B: Condensed Matter, vol. 302-303, pp. 140-148, 2001.

[17] S. He, S. C. Xin, L. J. Shan, L. B. Hui, Z. Z. Zhong, and S. D. Zhen, "Stable p-type $\mathrm{ZnO}$ films obtained by lithium-nitrogen codoping method," Physica Status Solidi B, vol. 250, no. 10, pp. 2102-2105, 2013.

[18] L. Meng, J. Zhang, J. An, and X. Hou, "Stable p-type ZnO thin films on sapphire and n-type $4 \mathrm{H}-\mathrm{SiC}$ achieved by controlling oxygen pressure using radical-source laser molecular beam epitaxy," Physica Status Solidi (A), vol. 213, no. 1, pp. 72-78, 2016.

[19] J. C. Fan, K. M. Sreekanth, Z. Xie, S. L. Chang, and K. V. Rao, "P-Type $\mathrm{ZnO}$ materials: theory, growth, properties and devices," Progress in Materials Science, vol. 58, no. 6, pp. 874-985, 2013.

[20] I. Takahashi, H. Hashimoto, Y. Fujita, and Y. Cho, "Measurement of distribution of P-type $\mathrm{ZnO}$ nano-particles by scanning nonlinear dielectric microscopy," in Proceedings of the 21st International Colloquium on Scanning Probe Microscopy, 8PN89, Tsukuba, Japan, November 2013.

[21] Y. Fujita, K. Moriyama, Y. Hiragino, Y. Furubayashi, H. Hashimoto, and T. Yoshida, "Electroluminescence from nitrogen doped $\mathrm{ZnO}$ nanoparticles," Physica Status Solidi (C), vol. 11, no. 7-8, pp. 1260-1262, 2014.

[22] D. C. Look, D. C. Reynolds, C. W. Litton, R. L. Jones, D. B. Eason, and G. Cantwell, "Characterization of homoepitaxial $p$ type $\mathrm{ZnO}$ grown by molecular beam epitaxy," Applied Physics Letters, vol. 81, no. 10, pp. 1830-1832, 2002.

[23] M. L. Tu, Y. K. Su, and C. Y. Ma, "Nitrogen-doped p-type ZnO films prepared from nitrogen gas radio-frequency magnetron sputtering," Journal of Applied Physics, vol. 100, no. 5, Article ID 053705, 2006.

[24] N. Y. Garces, N. C. Giles, L. E. Halliburton et al., "Production of nitrogen acceptors in $\mathrm{ZnO}$ by thermal annealing," Applied Physics Letters, vol. 80, no. 8, pp. 1334-1336, 2002.

[25] X.-L. Guo, H. Tabata, and T. Kawai, "Pulsed laser reactive deposition of p-type $\mathrm{ZnO}$ film enhanced by an electron cyclotron resonance source," Journal of Crystal Growth, vol. 223, no. 1-2, pp. 135-139, 2001.

[26] T. Varga, L. Kovarik, R. Sanghavi, V. Shutthanandan, and S. Thevuthasan, "Formation of zinc oxide films using submicron zinc particle dispersions," Journal of Vacuum Science \& Technology A, vol. 30, no. 4, Article ID 041805, 2012.

[27] N. Mechau, S. Bubel, D. Nikolova, and H. Hahn, "Influence of stabilizers in $\mathrm{ZnO}$ nano-dispersions on the performance of solution-processed FETs," Physica Status Solidi A, vol. 207, no. 7, pp. 1684-1688, 2010.

[28] S. Walther, S. Polster, M. P. M. Jank, H. Thiem, H. Ryssel, and L. Frey, "Tuning of charge carrier density of $\mathrm{ZnO}$ nanoparticle films by oxygen plasma treatment," Advanced Powder Technology, vol. 22, no. 2, pp. 253-256, 2011.

[29] M. S. Rajachidambaram, T. Varga, L. Kovarik et al., "Formation of zinc oxide films using submicron zinc particle dispersions," Journal of Vacuum Science and Technology B: Nanotechnology and Microelectronics, vol. 30, no. 4, Article ID 041805, 2012.

[30] B. Wang, J. H. Min, Y. Zhao, W. B. Sang, and C. J. Wang, "The grain boundary related $p$-type conductivity in $\mathrm{ZnO}$ films prepared by ultrasonic spray pyrolysis," Applied Physics Letters, vol. 94, no. 19, Article ID 192101, 2009.
[31] T. Yoshida, K. Shinohara, D. Itohara, and Y. Fujita, "Effects of thermal pressing on $\mathrm{ZnO}$ nanoparticle layers deposited by drop casting," e-Journal of Surface Science and Nanotechnology, vol. 14, pp. 175-178, 2016.

[32] S. Lee, D. Zhitomirsky, and J. C. Grossman, "Manipulating electronic energy disorder in colloidal quantum dot solids for enhanced charge carrier transport," Advanced Functional Materials, vol. 26, no. 10, pp. 1554-1562, 2016. 

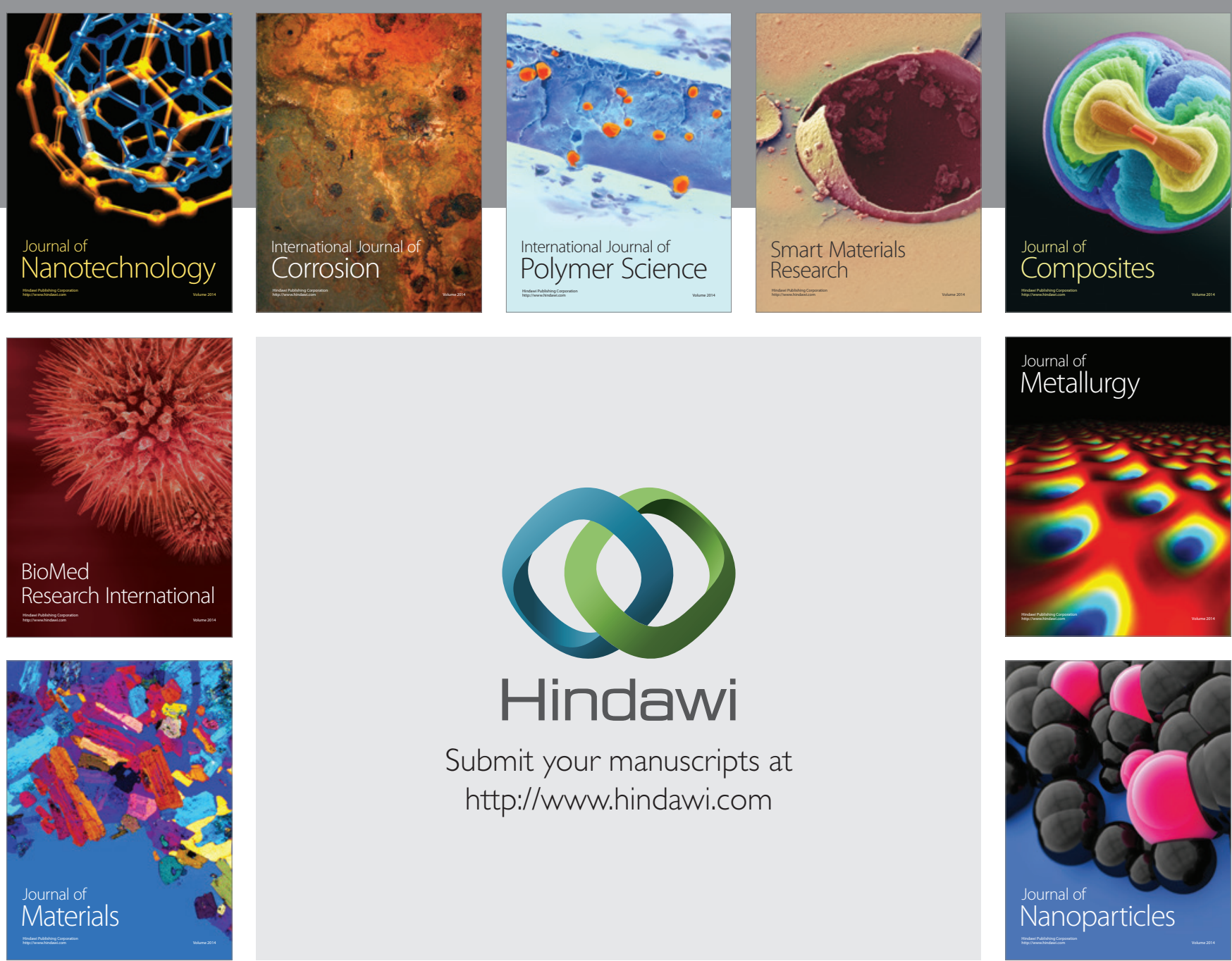

\section{Hindawi}

Submit your manuscripts at

http://www.hindawi.com

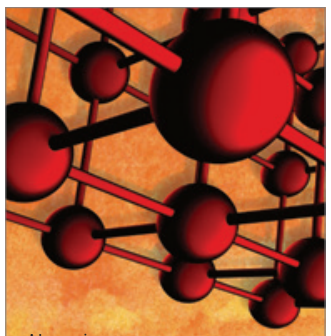

Materials Science and Engineering
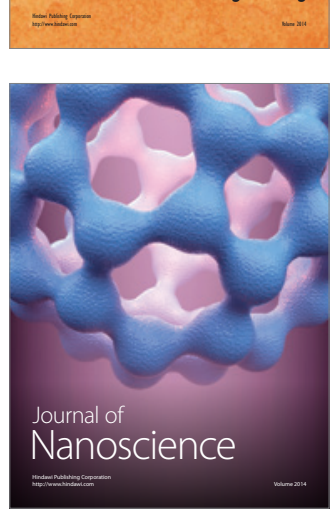
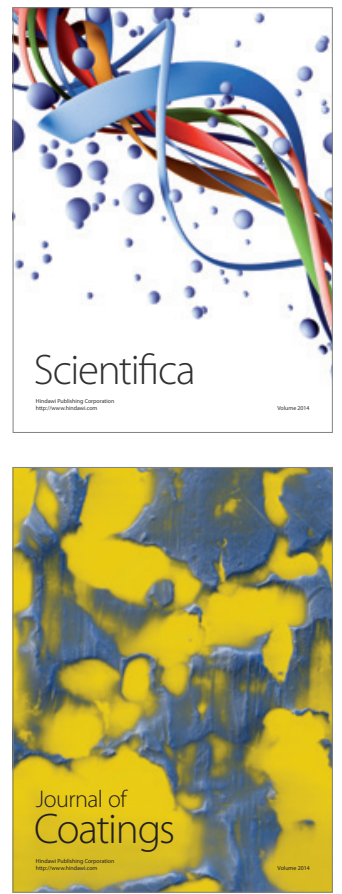
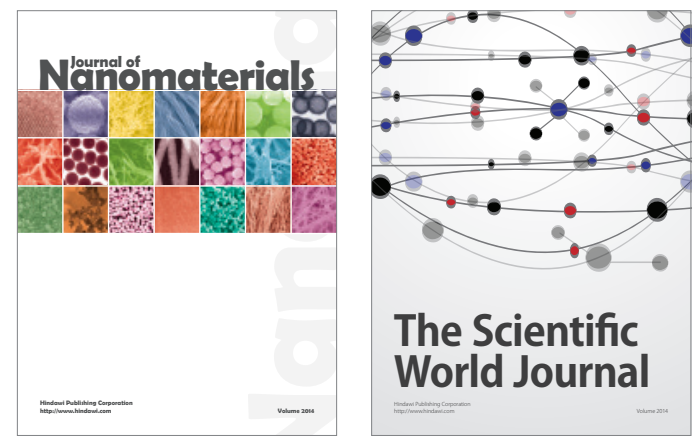

The Scientific World Journal
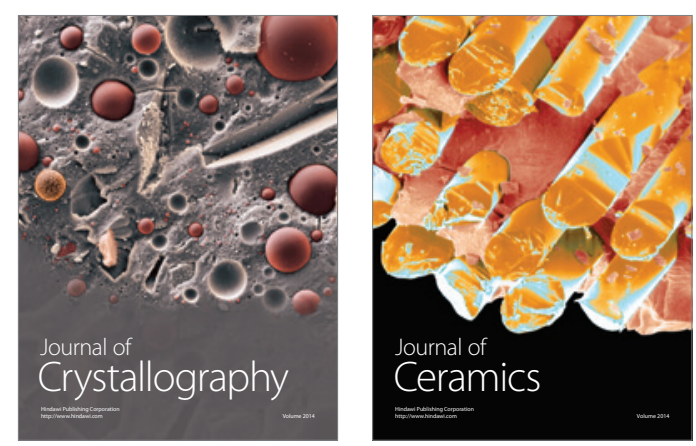
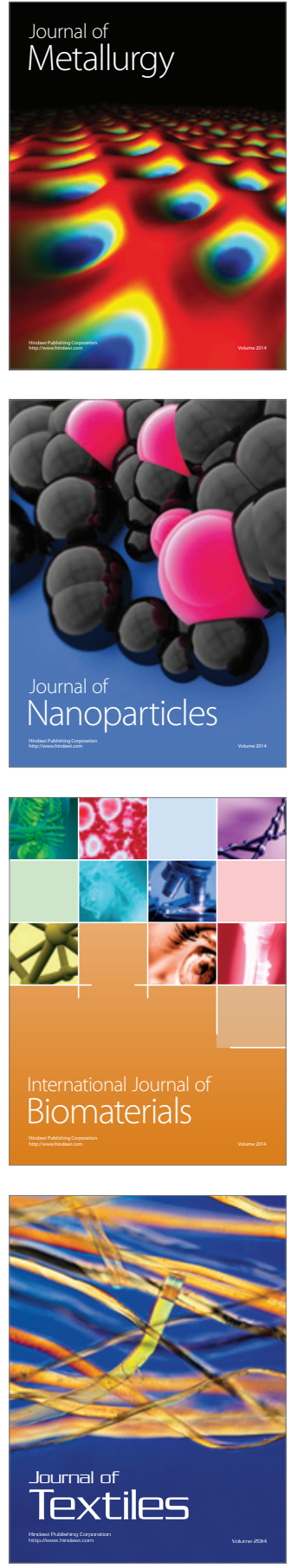